IdeAs

Idées d'Amériques

$10 \mid 2017$

États-Unis / Cuba : une nouvelle donne?

\title{
Cuba face aux soubresauts de la normalisation des relations avec les Etats-Unis
}

Cuba and the ups and downs of the normalization process with the United States Cuba frente a los sobresaldos de la normalización de las relaciones con los Estados Unidos

Janette Habel

\section{OpenEdition}

Journals

Édition électronique

URL : https://journals.openedition.org/ideas/2231

DOI : 10.4000/ideas.2231

ISSN : 1950-5701

Éditeur

Institut des Amériques

Référence électronique

Janette Habel, "Cuba face aux soubresauts de la normalisation des relations avec les Etats-Unis », IdeAs [En ligne], 10 | 2017, mis en ligne le 19 décembre 2017, consulté le 18 octobre 2022. URL : http:// journals.openedition.org/ideas/2231; DOI : https://doi.org/10.4000/ideas.2231

Ce document a été généré automatiquement le 18 octobre 2022

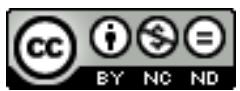

Creative Commons - Attribution - Pas d'Utilisation Commerciale - Pas de Modification 4.0 International - CC BY-NC-ND 4.0

https://creativecommons.org/licenses/by-nc-nd/4.0/ 


\section{Cuba face aux soubresauts de la normalisation des relations avec les Etats-Unis}

Cuba and the ups and downs of the normalization process with the United States Cuba frente a los sobresaldos de la normalización de las relaciones con los Estados Unidos

Janette Habel

\section{Introduction}

$1 \quad$ Normaliser des relations marquées par la confrontation entre leurs deux pays depuis un siècle et demi: tel est le défi que le président américain Barack Obama et son homologue cubain Raoul Castro, tous deux en fin de mandat, ont tenté de relever. Ce rapprochement que la géopolitique semblait imposer ne va pas de soi. Pendant plus d'un demi-siècle l'administration américaine a tenté d'effacer un affront historique et d'administrer une leçon aux peuples latino-américains. La crise économique cubaine devait mettre en évidence les échecs du castrisme et, grâce à une guerre d'usure économique et commerciale, provoquer un changement de régime politique, une stratégie s'inscrivant dans la politique du nation building. L'insuccès ou plutôt le fiasco de cette démarche avait amené Barack Obama à rechercher une voie diplomatique, en négociant un rapprochement sans préalable politique.

Du côté cubain, il est possible d'identifier les motivations qui ont poussé le président Raoul Castro, une fois le préalable politique levé, à initier un dialogue avec le président américain Obama. Dialogue risqué dans la mesure où Raoul Castro a engagé depuis 2011 un processus de réformes économiques qui se heurte à de nombreux obstacles sociaux et politiques et dont les résultats ne sont pas au rendez-vous.

3 Pour les dirigeants cubains, les déterminants économiques, sociaux et politiques incitent fortement à normaliser les rapports entre les deux pays. En outre, 
l'environnement régional a changé et les alliés politiques et partenaires économiques de Cuba, notamment le Brésil et surtout le Venezuela, sont en crise.

4 Le conflit séculaire avec les Etats-Unis a laissé des traces profondes dans la société cubaine. La crainte d'un retour au passé, le souvenir des humiliations subies sont encore présents. Depuis les guerres d'indépendance, la fierté nationale, souvent sousestimée par les observateurs, est un ingrédient de la résistance aux sanctions économiques imposées par Washington. Elle explique aussi l'anomalie représentée par un demi-siècle de conflictualité et d'absence de relations diplomatiques entre deux pays séparés par seulement $200 \mathrm{kms}$, " 90 miles from home » disent les Américains. Fidel Castro et son frère Raoul ont toujours conditionné la normalisation des relations diplomatiques avec les Etats Unis au respect par Washington de leur souveraineté, à la non-ingérence dans les affaires intérieures du pays, au refus de toute imposition concernant leur système politique. Ils se sont toujours méfiés du «néo conservatisme " des penseurs américains, "une doctrine qui consiste à penser que la démocratie libérale s'imposera partout dans le monde comme un 'métaprojet' » comme l'écrit Christian Lequesne (2017).

\section{Les raisons cubaines}

5 Pourquoi Raoul Castro a-t-il accepté de rentrer dans une longue négociation avec le président Obama ? Les raisons de cet engagement sont multiples. La toute première tient à la reconnaissance par le président américain des échecs passés et à l'abandon de toute condition préalable à la négociation. A la différence de la Loi Helms-Burton ${ }^{1}$ signée par le président Bill Clinton en 1996 et de la Commission d'aide à un Cuba libre ${ }^{2}$ créée par l'administration de George W. Bush, la Maison-Blanche n'a pas fait du départ des «frères Castro » une condition du rapprochement entre les deux pays ${ }^{3}$. Ce changement a provoqué la fureur contre Barack Obama des exilés cubains les plus conservateurs qui ont toujours exigé un changement de régime. En contrepartie de son engagement dans les négociations, La Havane espérait pouvoir obtenir à terme la levée de l'embargo, et comptait sur l'accroissement du tourisme nord-américain.

Barack Obama avait compris qu' il fallait renoncer à la politique du gros bâton (big stick) et emprunter la voie du dialogue en mettant à profit une conjoncture particulièrement difficile pour le gouvernement cubain. Il pensait pouvoir ainsi provoquer par d'autres voies le changement politique souhaité. Une tactique comprise par Fidel Castro qui fit part de sa défiance dans un éditorial publié après la visite de Barack Obama à La Havane en mars 2016. Ce dernier avait en effet expliqué que sa visite lui laissait entrevoir ce "que nous pourrions faire ensemble comme amis, comme membres d'une même famille, comme voisins, tous ensemble». Intitulé "Le frère Obama» l'article de Fidel Castro mettait en garde contre les illusions suscitées par le discours du visiteur. « Nous avons failli faire un infarctus en écoutant ces paroles du président des Etats-Unis. Nous sommes capables de produire les aliments et les richesses dont nous avons besoin grâce aux efforts et à l'intelligence de notre peuple. Nous n'avons pas besoin des cadeaux de l'empire » écrivait-il (Castro F., 2016).

Décédé le 25 novembre 2016, 17 jours après l'élection de Donald Trump, Fidel Castro n'aura pas assisté au retour du bâton.

7 L'année 2017 aura été difficile pour La Havane. Commencée l'hiver avec l'élection surprise de Donald Trump, elle s'est achevée à l'automne dans la tourmente provoquée 
par un cyclone d'une force exceptionnelle, à cinq mois d'une échéance électorale et politique majeure. En février 2018, cinquante ans après la Révolution, pour la première fois, Cuba ne devrait plus avoir un Castro comme président. Pendant 47 ans Fidel Castro a gouverné le pays. En 2006, sa maladie l'obligea à interrompre ses activités, provisoirement d'abord, puis définitivement, en officialisant en 2008 la passation des pouvoirs à son frère cadet, Raoul Castro, ministre des Forces Armées et deuxième secrétaire du Parti communiste. Raoul Castro aura gouverné pendant douze ans. Mais si «Fidélisme » et « Raoulisme 4 » ont marqué l'histoire cubaine pendant près de soixante ans, le cours politique suivi pendant plus d'une décennie par Raoul Castro représente une inflexion substantielle à l'égard de la stratégie économique impulsée précédemment par son frère aîné . C'est sous sa présidence que le rapprochement spectaculaire entre les deux Etats a été initié secrètement, puis rendu public en 2014, cherchant à inaugurer une ère nouvelle entre les deux pays. Ce début de normalisation impliquait des avancées et des contreparties de part et d'autre. Un rapprochement susceptible d'ouvrir une brèche dans un embargo vieux d'un demi siècle était une opportunité pour La Havane compte tenu des difficultés économiques et financières du pays, aggravées par la crise financière mondiale de 2008. «Les politiques économiques de survie se sont aggravées dans notre pays et elles mettent en danger notre stabilité et notre identité nationale » constate un jeune cubain (López Inguanzo J.M., 2017).

Du côté américain, quelles étaient les raisons ayant conduit à ce processus ? Outre la recherche par Obama d'un marqueur historique pour sa présidence, plusieurs raisons contribuaient à cette initiative. Les réformes, la libéralisation et l'ouverture économiques impulsées dans l'île par Raoul Castro depuis 2011 devaient faciliter les échanges commerciaux et les investissements . Barack Obama, adepte du «soft power $^{5}$ ", avait compris qu'elles permettraient aussi d'accroitre l'influence politique et culturelle de l' "American way of life » dans une société marquée par de nombreuses privations. Autre élément, la réorientation pragmatique de la diplomatie cubaine en Amérique latine, dont témoignait sa contribution aux négociations entre les Forces Armées Révolutionnaires Colombiennes (FARC) et le gouvernement conservateur colombien de Juan Manuel Santos. Enfin, Barack Obama était confronté à brève échéance à un enjeu diplomatique continental, la tenue du Sommet des Amériques en avril 2015 au Panama dont La Havane était exclue. Depuis plusieurs années, de nombreux gouvernants latino-américains y compris conservateurs, menaçaient de boycotter cette réunion si Cuba n'y participait pas. En 2015 le président américain risquait de se trouver face à des chaises vides. Après l'annonce conjointe du rapprochement des deux Etats en décembre 2014 le succès du Sommet continental était garanti pour la Maison Blanche.

\section{Retour sur l'embargo}

Outre leurs effets économiques, commerciaux, financiers, les sanctions ont eu des conséquences néfastes sur le plan politique, démocratique et culturel. Il n'est pas superflu de prendre la mesure de cette anomalie : un embargo imposé pendant plus d'un demi-siècle ${ }^{6}$, dont l'efficacité est renforcée par la proximité géographique de la première puissance mondiale et la fermeture d'un marché qui a représenté le débouché historique naturel et presque unique du commerce cubain pendant des décennies 
(Moreno Fraginals M., 1978). Avant 1959 les deux tiers des échanges de l'île se faisaient avec les Etats-Unis.

«L'embargo est l'obstacle le plus important pour notre développement économique » avait déclaré Raoul Castro. La législation américaine dissuade des banques et de nombreuses entreprises étrangères de s'installer à Cuba de peur des représailles américaines, des pénalités financières et de l'augmentation des coûts de transaction.

Dans un rapport d'information déposé par la Commission des Affaires Etrangères et la Commission des finances de l'Assemblée Nationale sur l'extraterritorialité de la législation américaine, le président Pierre Lellouche et la rapporteure Karine Berger soulignent sa nocivité sous- estimée (Assemblée nationale, 2016). Ils dénoncent «les amendes colossales infligées par les Etats-Unis à des entreprises européennes. Les entreprises françaises ont particulièrement été frappées en 2014, année qui a vu la banque BNP-Paribas accepter de payer un montant record de près de 9 milliards de dollars pour avoir violé les embargos financiers des Etats-Unis contre plusieurs pays » (en particulier Cuba). "L'Office of Foreign Assets Control (OFAC ), service du Trésor qui veille à l'application des sanctions internationales américaines dans le domaine financier, emploie environ 200 personnes et a un budget de plus de 30 millions de dollars ». . « Ainsi le droit est mis au service des objectifs de la politique étrangère et des intérêts économiques des Etats-Unis » soulignent les rapporteurs. La loi américaine dite Helms-Burton ouvre des facultés de rétorsion de caractère extraterritorial. Elle prétend sanctionner (par des poursuites civiles aux Etats-Unis et l'interdiction d'entrer sur leur territoire) les personnes et entreprises de toute nationalité 'trafiquant' (achetant, louant, prenant en gestion...) des biens confisqués par le gouvernement cubain à des citoyens américains (y compris les exilés cubains devenus ensuite américains). Une ingérence expresse dans le gouvernement d'un pays étranger à l'encontre de principes du droit international » concluent-ils.

Le diplomate Howlett-Martin (2017) rappelle que l'OFAC a interdit l'importation aux Etats-Unis d'équipements contenant du nickel en provenance de Cuba, de chocolat suisse fabriqué à partir de cacao cubain, il a fait saisir en janvier 2011 la contribution destinée à Cuba du Fonds mondial de lutte contre le sida, la tuberculose et le paludisme (Global Fund) d'un montant de 4,2 millions de dollars.

13 Quelle définition faut-il donner de ces sanctions? La polémique terminologique oppose la définition cubaine 'bloqueo' (blocus) à la française (embargo). "C'est un blocus embargo, c'est moins qu'un blocus mais plus qu'un embargo ", car "il empêche des tiers de commercer avec Cuba" précise l'ancien ambassadeur de France Jean Mendelson (2017).

Pour Cuba les raisons du rapprochement ne sont pas seulement économiques, commerciales et financières. Des contraintes politiques, sociales et générationnelles, régionales et internationales rendent nécessaire la normalisation avec les Etats Unis, une normalisation facilitée par l'ouverture économique en cours. Mais le pays est fragilisé, l'île est entrée dans un "quinquennat de transition " (Raoul Castro) difficile. Pour la direction actuelle, la transition en cours est un processus politique trop délicat pour en laisser la seule responsabilité aux successeurs. C'est pourquoi Raoul Castro avait saisi l'opportunité offerte par l'administration Obama pour engager de son vivant une négociation à hauts risques. 


\section{Les défis de l' « actualisation du modèle »}

15 Le constat est presque général : le "modèle » économique est à bout de souffle. Sept ans ont passé depuis l'avertissement de Raoul Castro en décembre 2010 : «Le temps où l'on pouvait marcher au bord du précipice est fini. Soit nous rectifions, soit nous sombrons et l'effort de générations entières sombrera avec nous ». La révision du «modèle » est à l'ordre du jour. Les réformes engagées par Raoul Castro donnent plus d'importance au secteur privé, diminuent le poids de l'Etat et des dépenses sociales jugées insoutenables. La nouvelle loi sur les investissements étrangers adoptée en 2014 offre des avantages fiscaux importants, dont l'exonération pendant huit ans de l'impôt sur les bénéfices. Car les difficultés économiques perdurent, aggravées par les crises vénézuélienne et brésilienne. Cuba est donc confrontée à la crise de ses principaux alliés latino-américains et au revirement de l'imprévisible président américain Donald Trump.

16 Mais le rythme des réformes fait débat. Trop lentes pour les uns, elles vont trop vite pour les autres. Des prises de positions qui recouvrent des enjeux sociopolitiques. Bien que partielles, les réformes ont déjà provoqué une croissance significative des inégalités qui affaiblit la base sociale du régime. Les difficultés de la vie quotidienne suscitent des insatisfactions, la précarité s'étend, la loyauté envers le régime s'affaiblit. Viennent s'ajouter les transformations de la société cubaine induites par la libéralisation du secteur marchand. En 2015, 70,8\% des salariés travaillaient encore pour l'Etat au lieu de $98 \%$ avant 1992. Les auto-entrepreneurs et les petites entreprises représentent désormais $30 \%$ de la population active, ce qui implique une autonomie personnelle et politique accrue à l'égard du pouvoir. La société cubaine est aujourd'hui beaucoup plus hétérogène, plus diversifiée. Alors que l'ancien ministre et président de la Junta Central de Planificación Humberto Perez (2016) souligne "la faiblesse que représente le fait que notre société soit déchirée et fracturée en différents segments inégaux économiquement et socialement avec une majorité de la population - ouvriers et employés du secteur d'Etat, retraités - qui ne perçoivent pas des revenus légaux ${ }^{7}$ suffisants pour vivre», la sociologue Daybel Panellas analyse (2015) «l'autre pôle de l'inégalité : les nouveaux riches, les gérants. Les réformes n'apportent des bénéfices que pour ceux qui occupent des positions privilégiées, l'argent s'impose comme une bannière permettant de se positionner socialement, d'établir des relations ".

17 Fidel Castro défendait une conception idéologique de l'homogénéité politico-culturelle $\mathrm{du}$ peuple et refusait les divisions sociales, raciales ou communautaires au nom de l'égalité et de l'unité nationale indispensables. Aujourd'hui, la diversité socioéconomique fragilise un leadership peu habitué à gérer cette nouvelle fragmentation. Plus important encore, les générations qui n'ont pas connu la période prérévolutionnaire et qui sont nées avec la crise aspirent aux échanges, aux voyages, à l'information, aux nouvelles technologies, à Internet, à ce qui leur apparaît comme la modernité , une histoire contemporaine connectée. Ils voient la société à travers une multitude de groupes éclatés, souvent sans perspective. Humberto Perez fait un constat lucide : « Il faut tenir compte du fait que la majorité de la génération actuelle est née et a grandi au milieu des avatars et des pénuries de la 'période spéciale', 60 ans après le capitalisme, avec une lecture différente de notre processus révolutionnaire, en partie par ignorance ou par oubli, en partie à cause des écarts générationnels. Il y a une accumulation de besoins insatisfaits, d'attentes et d'aspirations régulièrement 
frustrées, alors que leur niveau culturel moyen est élevé, ce qui renforce leur exigence critique ». La société cubaine a rarement été aussi vulnérable.

Le débat économique n'est pas nouveau à Cuba. En 1996, les économistes Pedro Monreal et Julio Carranza avaient déjà évoqué - en vain - la nécessité d'une "restructuration économique " (Carranza J. et al, 1996). Aujourd'hui cette restructuration a commencé sous le vocable d'« actualisation ». La discussion est vive, elle porte sur l'ampleur et les rythmes d'application de la restructuration compte tenu des résistances sociales et politiques qu'elle rencontre. Comment et jusqu'où "actualiser le modèle économique » sans mettre en cause la stabilité politique et sociale? Quelle transition faut-il mettre en œuvre dans une conjoncture complexe que les destructions provoquées par l'ouragan Irma ont encore aggravée ? Ce sont les populations les plus pauvres qui ont le plus souffert, aiguisant les tensions sociales. En témoigne une alerte peu habituelle à La Havane où des habitants du quartier 10 de octubre se sont rassemblés dans la rue après le passage du cyclone pour protester contre le manque d'eau et d'électricité.

C'est dans ce contexte socio-politique très sensible que le débat sur la " conceptualisation du modèle économique et social ${ }^{8}$ " s'est engagé avant même sa ratification par le Comité Central du PCC. Ce texte définit les bases essentielles destinées à «consolider les principes socialistes du pays » sur le plan économique et social. L' " actualisation du modèle économique et social cubain » est présentée comme indispensable afin de "rendre irréversible la construction du socialisme». Le parti communiste contrôle la mise en place de cette rénovation. Le rôle primordial de la propriété sociale sur les principaux moyens de production, la reconnaissance de divers types de propriété et de gestion et le "perfectionnement de la gestion socialiste de l'Etat sont affirmés». Quatre formes de propriétés sont reconnues. «La propriété socialiste (dont les coopératives), la propriété mixte (entreprise étrangère en association avec une entreprise cubaine autorisée pour une période déterminée) , la propriété privée (personnes physiques ou morales, cubaines ou étrangères dans certaines activités autorisées au cas par cas pour les entreprises étrangères, et uniquement pour des petites ou moyennes entreprises pour les Cubains) et la propriété d'organisations associatives ".

Le débat longtemps feutré s'est développé chez les intellectuels et les économistes. Ces derniers divergent sur le rythme des réformes, sur les rapports entre la planification et le marché et sur la place du secteur privé. Comment éviter la concentration de la propriété et de la richesse dans le secteur non étatique ? Quelle est la dynamique de la transition en cours et de la démocratisation - ou pas- du système politique ? Pour le politologue Juan Valdès Paz_ "l'élément clé de ces réformes c'est la création d'une sphère où les entreprises sont réellement autonomes, basée sur la diversification des formes de propriété et de gestion, la diversité des agents économiques dans les différents secteurs de l'économie ${ }^{9}$ ». Désormais les analyses et le débat sont publics sur Internet ${ }^{10}$ et la liste des blogs est longue : Cuba Debate, El estado como tal, Cuba Posible, La Joven Cuba, Observatorio Crítico, Diario de Cuba, 14YMEDIO etc. Les échanges opposent d'anciens ministres, des cadres politiques du PCC, des intellectuels, des militants des différentes organisations populaires. Parmi les résolutions adoptées par le PCC et ratifiées par l'Assemblée nationale (ANPP), celle sur la «Conceptualisation du modèle économique et social cubain de développement socialiste" devait proposer une conception théorique générale et définir les rapports entre planification et marché. 
Mais le compromis intervenu entre les différentes positions brouille les cartes. Selon le point VI « la planification socialiste est la catégorie principale du système de direction, grâce à laquelle on élabore les objectifs à atteindre. Elle donne la priorité au développement stratégique, elle est centralisée, participative, et différenciée selon les instances ». Mais le point VII précise qu'il s'agit de «reconnaître, réguler, obtenir un fonctionnement adéquat du marché de sorte que les décisions administratives centralisées, en interaction avec les politiques macro-économiques et les autres, incitent les acteurs économiques à adopter des décisions en accord avec les intérêts de toute la société ». Comme on pouvait le prévoir, après l'adoption et la publication du texte, la discussion s'est poursuivie. L'accord est général sur le diagnostic de la gravité de la situation. Mais les avis divergent sur les solutions à apporter. Pour l'ancien ministre de l'Economie José Luis Rodriguez (2017), « Il ne faut pas perdre de vue que la planification a un potentiel très supérieur au marché pour parvenir au développement, comme le montrent les exemples de certains pays asiatiques tels que Singapour ou la Corée du Sud. Une planification plus flexible, qui tienne compte du marché, qui s'appuie pour son élaboration sur la participation populaire, devrait obtenir des résultats beaucoup plus efficaces pour l'économie». Oscar Fernández, un autre économiste, résume ainsi sa position : «le défi de Cuba, c'est d'étendre le marché et de développer la planification ${ }^{11} »$. Mais que faut-il planifier et jusqu'où? Agustín Lage, ancien membre du Comité central et dirigeant du Centre d'immunologie moléculaire (CIM) est intervenu pour souligner la nécessité d'un budget planifié pour l'activité scientifique, alors qu'il est proposé qu'elle soit financée par les bénéfices commerciaux des entreprises, en particulier par BioCubaFarma ${ }^{12}$. Cette demande reçut une réponse réservée et dilatoire de la part de la ministre.

Le nouveau modèle économique suppose une autre conception du système de direction et de planification de l'économie. L'embauche de la force de travail par des agents privés est désormais autorisée, elle crée les prémisses d'un marché du travail. Pour le politologue Juan Valdès, l'embauche de salariés par le secteur privé va changer profondément le modèle économique en vigueur. De fait, toutes les réformes modifient la politique et le marché de l'emploi. En mars 2014, un nouveau Code du Travail a été approuvé, il définit les droits des salariés des petits entrepreneurs privés. Mais pour le juriste Julio Antonio Fernandez Estrada (2017), «ceux-ci sont évoqués de façon générique dans le Code du Travail mais il n'est pas précisé de régime spécifique pour les salariés des entrepreneurs privés. Il n'est pas spécifié que les contrats de travail ne peuvent être oraux, que le salaire minimum doit être indiqué, de même que le paiement des vacances et les horaires de travail ».

Humberto Perez (2016), président du Conseil central de planification de 1976 à 1985, estime qu'il y a urgence : il s'agit de «prendre les mesures adéquates pour sauver le caractère socialiste de notre processus pour lequel nous avons tant lutté et que nous risquons de perdre ». Mais le contenu réel de ce caractère "socialiste» ne fait pas l'unanimité. Officiellement le concept est maintenu. En réalité, pour certains partisans des réformes le seul objectif réaliste possible est la défense de l'indépendance nationale, ils contestent la lenteur des réformes, défendue par Raoul Castro, justifiée par des dirigeants du PCC au nom de la sauvegarde du socialisme. "On peut être d'accord sur le fait que le socialisme et le capitalisme ne sont pas compatibles " commente l'économiste Julio Carranza (2017) «mais je ne crois pas que nous soyons d'accord sur ce qu'est le socialisme ». 

possible, il n'a pas de possibilité de capitalisme cubain autonome, ce serait un capitalisme "nord-américano-cubain». L'alternative ne peut être d'introduire des éléments du capitalisme en pensant que l'on va faire ce que d'autres dans le passé ont appelé un socialisme d'Etat, autrement dit une transition permettant à une quantité de fonctionnaires de se transformer en entrepreneurs. Ceci n'est pas faisable à Cuba. A Cuba, on ne peut pas dire "on va construire le capitalisme, mais ce ne sera pas un capitalisme néo libéral, vous verrez notre politique sociale sera très bonne. Tout cela n'est pas possible".

De nombreux économistes réformateurs ont analysé les réformes chinoise (1978) et vietnamienne (1986) et notamment le rôle de la diaspora dans la dynamique économique initiale. Ils savent que pour «actualiser » le modèle économique et social, le rendre " prospère et soutenable » selon les termes de Raoul Castro, les ressources, les capitaux (ceux de la diaspora compris), les investissements étrangers sont indispensables.

\section{La diaspora, pomme de discorde}

La libéralisation et l'ouverture économiques devraient permettre de mettre à profit les ressources financières de la diaspora cubaine, comme l'ont fait la Chine et le Vietnam qui ont su utiliser le potentiel financier de leur émigration. La diaspora cubaine est à la fois une des solutions à la crise mais elle est aussi une source de tensions. L'émigration cubaine aux Etats- Unis n'est comparable ni à la chinoise ni à la vietnamienne. Pour comprendre son caractère exceptionnel, il faut revisiter l'histoire et la géographie. Des liens historiques unissent la Floride et Cuba, séparés seulement par une étendue maritime de $200 \mathrm{kms}$. Mais après 1959 ces exilés étaient en territoire " ennemi » et ont été traités comme tels pendant de longues années, Fidel Castro les avait surnommés les gusanos (les vers de terre). L'effondrement de l'URSS a changé la donne. Les élites cubano- américaines issues de la première immigration, celle de la grande bourgeoisie, ont construit en cinq décennies la plus grande concentration cubaine à l'extérieur de l'île. Elles ont « latinisé » Miami, elles en ont fait une « ville carrefour» (Jolivet V., 2015) entre l'Amérique du Nord et celle du Sud, un centre d'affaires où se négocient des transactions financières internationales, elles y ont construit un empire immobilier. Leur puissance financière leur a permis de former un lobby très influent au sein de l'administration américaine faisant de la question cubaine un problème de politique intérieure. Elles exercent aussi un pouvoir et un contrôle politique important sur les 1 500000 Cubains arrivés au fil des ans qui vivent sur ce territoire. Le rapport de cette " troisième Amérique ${ }^{13}$ » et de ses élites avec leur pays d'origine n'a cessé d'être conflictuel. Dans les familles déchirées, l'histoire croisée oppose récits et contre-récits, des mémoires et des représentations contradictoires dont témoignent les deux monuments célébrant l'expédition de la Baie des Cochons en 1961 érigés l'un à Miami, en l'honneur des vaincus de l'expédition, et l'autre à La Havane célébrant la victoire de la résistance populaire sur les envahisseurs.

Nécessité faisant loi, La Havane a multiplié ces dernières années les tentatives pour diviser politiquement la diaspora. Les émigrés les plus récents diffèrent en effet de ceux qui les ont précédés : leurs motivations sont moins politiques et plus économiques, ils n'adhèrent pas aux propositions agressives des représentants de la Cuban American 
National Fondation (CANF) comme le montre l'appui dont a bénéficié la politique de Barack Obama lors de l'élection présidentielle. Comme le constate l'ancien ambassadeur Jean Mendelson (2017), « on assiste au sein de l'exil à un changement générationnel. Il y a ceux qui sont partis au début de la révolution, qui ont haï toute leur vie «les frères Castro » et qui font penser souvent aux émigrés de la Révolution française rentrés au bout de 25 ans sans avoir 'rien appris ni rien oublié'. Dans le cas cubain cela fera bientôt soixante ans, et cette opposition est en voie de disparition ». Selon les résultats d'un sondage publié par l'université de Miami, "la majorité des exilés souhaitent une plus grande ouverture vers leur patrie d'origine et se prononcent contre l'embargo. En 1991 ils n'étaient que 13\% contre 22\% en 1997, 34\% en 2004, 46\% en 2011 et $52 \%$ en $2014^{14}$.

Mais le poids politique des élus cubano-américains - républicains surtout mais aussi démocrates dans une moindre mesure - reste encore considérable. Sous l'influence de Marco Rubio, ancien candidat républicain à l'élection présidentielle et sénateur de Floride, le président Donald Trump a remis en cause le rapprochement impulsé par son prédécesseur. Ce contexte politique est d'autant plus sensible dans l'île qu'une partie de la population perçoit avec amertume la réussite et l'aisance matérielle de ceux qui étaient partis et qui reviennent, même temporairement.

D'ores et déjà l'impact des ressources financières provenant de l'émigration se fait sentir. D'après José Luis Rodriguez, ancien ministre de l'Economie, « les envois d'argent sont en hausse, ils atteignent environ 3 milliards de dollars par an ", une somme encore supérieure selon certains. On estime qu'environ $50 \%$ des envois sont investis par leurs récepteurs et les autres $50 \%$ sont destinés à la consommation ». Il faut ajouter à ces envois les revenus directs en CUC ou en devises qui proviennent des locations de chambres dans les maisons ou les appartements privés (on estime qu'il y en a au moins 20.000), les gains des petits restaurants privés (paladars), des chauffeurs de taxis et autres auto entrepreneurs qui vendent leurs services directement aux étrangers, sans oublier les pourboires et les gratifications dont bénéficient ceux qui travaillent dans des entreprises étrangères, les ventes de maisons à des étrangers, les revenus des " escort girls » etc. Autant de facteurs qui exercent une pression sur les prix très élevés des marchés, notamment des produits agricoles où la population s'approvisionne ». Et Jose Luis Rodriguez (juillet 2017) de conclure «En pratique c'est comme si on ouvrait les portes de notre pays à une population qui réside dans un autre, et qui vient acheter sur nos marchés grâce aux revenus qu'elle a gagnés dans un autre pays, et dont le montant dépasse la demande solvable de la population de l'île ». Ces composantes exogènes concernent une minorité, environ 20 à $25 \%$ de la population, mais ces secteurs ont la capacité de payer leurs achats à des prix très élevés et d'absorber ainsi une offre de produits très limitée. L'importance de cette aide extérieure est manifeste dans la vie quotidienne, notamment pour les communications téléphoniques. Lorsqu'elles sont interrompues, ce sont les familles à l'extérieur du pays qui règlent la facture afin de pouvoir rester connectées.

\section{Le débat politique et historique}

29 Autre sujet en discussion: les changements institutionnels et politiques doivent ils accompagner les réformes économiques? La mort de F. Castro et la fin prochaine de la génération révolutionnaire mettent à l'ordre du jour le bilan historique et les nouvelles 
perspectives stratégiques. La critique politique est aujourd'hui publique même si elle est surtout réservée à ceux qui ont accès à Internet. "Il faut éliminer les comportements autoritaires. Le système doit promouvoir la discussion, le débat, impulser la différence y compris les divergences » écrivait Martinez Heredia (2017). Le débat est croisé. Les analyses et les prises de position ne sont pas homogènes. Certains dirigeants du PCC font valoir que, face à la politique agressive engagée par Donald Trump, l'unité est incontournable, il ne faut pas donner des armes à l'ennemi en se divisant. Un argument repris par Raoul Castro (2011) : «Cuba fait partie des quelques pays dans le monde qui peuvent transformer leur modèle économique et sortir de la crise sans traumatismes sociaux parce que nous avons un peuple patriotique, puissant, grâce à la force que représente son unité monolithique, la justesse de sa cause et sa préparation militaire».

30 Mais il n'est pas sûr que cette « unité monolithique » déjà préconisée par Fidel Castro à l'époque de la guerre froide fasse consensus aujourd'hui. "Le modèle social paternaliste, le contrôle et la surveillance ne sont pas possibles dans un pays éduqué " (Cárdenas H., 2017). L'héritage soviétique est de plus en plus contesté, en particulier par les nouvelles générations, mais la tradition nationaliste radicale et autoritaire héritée des guerres d'indépendance reste très vivace.

31 Pour comprendre les termes du débat il faut rappeler que si, pour les Cubains, la chute de l'URSS a été une catastrophe économique et commerciale et un traumatisme politique et idéologique majeur, aucune explication, aucune analyse officielle n'ont été données de cet effondrement. La glasnost et la perestroika étaient, il y a peu encore, des termes tabous. L'implosion soviétique fut comparée par Fidel Castro à « l'effondrement d'une meringue » (desmerengada) et présentée implicitement comme la conséquence des réformes de $\mathrm{M}$. Gorbatchev. Aller au-delà aurait supposé de faire le bilan du régime soviétique, de ses institutions, de ses échecs, mais une telle analyse aurait aussitôt imposé de dresser en parallèle un bilan de la politique cubaine. "Nous avons trop copié » affirmera Fidel Castro mais il n'en dira pas plus. Pour l'économiste Julio Carranza (juillet 2017), "ce silence représente un lourd handicap ", car "sans une discussion rigoureuse de l'expérience historique du socialisme réel, il est très difficile de parler sérieusement de son avenir où que ce soit, mais encore plus dans les conditions concrètes de Cuba. Parmi les débats nécessaires, il faut discuter des problèmes que le socialisme historique n'a jamais résolu de façon adéquate en quelque lieu que ce soit, notamment la question du marché et celle de la démocratie. Je ne peux que préciser ici que le socialisme doit garantir la souveraineté de tout le peuple, pas la souveraineté de la bureaucratie ». Une bureaucratie obsolète particulièrement contestée par les jeunes générations, souvent victimes de l'arbitraire, de l'autoritarisme voire des abus de pouvoir des fonctionnaires. Parfois anecdotiques, les exemples abondent de comportements qui frisent le ridicule, telle cette interdiction faite à une étudiante française en short de rentrer dans une bibliothèque au motif que son short était inconvenant ${ }^{15}$ (dans une île où la chaleur avoisine souvent les 30 degrés), ou plus grave, le renvoi de son poste universitaire d'un enseignant, Julio Antonio Fernández Estrada (Cárdenas H., janvier 2017). De nombreux blogs animés par des jeunes se font l'écho de ces pratiques et réclament des changements.

32 La démocratisation du système est considérée comme la condition de l'efficacité des réformes économiques, voire comme un contrepoids au marché par certains cadres politiques ou intellectuels mais ils sont peu nombreux. Ils rappellent que l'ouverture au 
marché n'est pas synonyme d'ouverture politique, ce que confirment les exemples chinois ou vietnamien. Pour le sociologue Juan Valdès Paz (2014) ${ }^{16}$, les changements institutionnels sont incontournables. Mais comment réformer le système sans affaiblir la base populaire de la révolution qui explique en grande partie la longévité du régime? Des questions qui divisent aussi bien les économistes, la bureaucratie, les militants, les intellectuels, sans oublier l'Eglise catholique ${ }^{17}$ dont le rôle , comme celui du Pape François, a été important dans la négociation avec l'administration Obama. L'Eglise est elle aussi partagée entre plusieurs positions. Certaines publications dirigées par des catholiques laïques telles que Cuba Posible, ont une perspective pluraliste et alimentent le débat politique en cours.

«Ce modèle économique impliquant des inégalités sociales plus importantes doit être complété par un développement plus grand de la démocratie. Les institutions devront être radicalement démocratisées et garantir des droits reconnus dans la Constitution : la participation des producteurs et des consommateurs au processus de planification, le contrôle du plan et des politiques économiques par la population grâce à un débat public et permanent, la participation directe des travailleurs à la gestion économique de leurs entreprises » affirme Juan Valdès Paz (2014).

L' " actualisation » devrait donc être le point de départ d'une réforme plus complète de l'ordre constitutionnel afin de donner une légitimité politique au nouveau modèle économique.

\section{8 : les contraintes politiques de la succession}

Pour le gouvernement, l'urgence est de concrétiser la dernière étape de la transition générationnelle en assurant une transition politique inédite, sans compromettre la stabilité du régime lorsque s'achèvera le mandat présidentiel de Raoul Castro en février 2018. Un pari que l'élection de Donald Trump a rendu plus difficile.

De nombreuses incertitudes demeurent concernant les modifications constitutionnelles. Elles sont débattues dans une Commission dont on ignore pour l'instant les conclusions finales. Mais le rôle dirigeant du Parti communiste cubain et sa suprématie dans la gestion des affaires de l'Etat seront réaffirmés. Le PCC est, avec les Forces Armées, le principal acteur du processus de réformes économiques ${ }^{18}$. Seules deux propositions sont connues : la première concerne la nécessité de séparer le PCC et la gestion étatique, administrative, jusqu'alors toujours confondus. Ce principe a déjà été réaffirmé dans le passé mais jamais appliqué. Il sera cette fois au moins formellement mis en œuvre puisque pour la première fois le président de la République et le premier secrétaire du PCC ne devraient plus être incarnés par la même personne. Miguel Diaz Canel, déjà premier vice-président, devrait remplacer Raoul Castro à la tête de l'Etat, ce dernier restant - mais pour combien de temps ?- premier secrétaire du PCC. Son successeur demeure jusqu'alors inconnu. La deuxième décision, annoncée par Raoul Castro, est significative: les postes clés de la direction politique et de l'Etat seront désormais limités à deux mandats de cinq ans. La succession ne concernera donc pas seulement Raoul Castro. Tous les cadres issus de la génération qui a combattu pour renverser la dictature de Fulgencio Batista en 1959 seront remplacés. D'autres propositions sont en discussion. La place des organisations populaires devrait être redéfinie pour qu'elles reflètent davantage la diversité de la société et soient plus autonomes. 

pressantes. « Nous entendons par là des normes et des institutions créées sur des bases démocratiques auxquelles sont subordonnées les institutions de l'Etat et de l'Administration publique " précise un interlocuteur. Lors d'un débat public un participant confirme :"A Cuba l'Etat de droit connaît de sérieuses difficultés ${ }^{19}$. Il faut protéger les droits des citoyens, dénoncer les comportements bureaucratiques y compris publiquement et les sanctionner civilement, et pénalement si nécessaire, ajoute un autre.

Pour le sociologue Juan Valdès, il ne fait pas de doute que «le nouveau modèle économique et la réforme constitutionnelle donneront lieu à une lutte idéologique intense entre les différents secteurs et courants politiques internes». Et de conclure : «Le socialisme ne peut retarder la démocratie qu'il a promise », une affirmation loin de faire consensus.

L'évolution des relations diplomatiques entre Cuba et les Etats Unis conditionnera fortement les changements politiques. L'élection de Donald Trump a déjà changé la donne. Pour le nouveau président américain, Cuba serait «un régime corrompu, déstabilisateur ». En déterrant la hache de guerre, Donald Trump renforce la conviction de la direction cubaine que la souveraineté nationale de l'île n'a jamais cessé d'être menacée. Celle-ci pourrait en conséquence chercher à resserrer les rangs, en contrôlant plus étroitement, et au besoin en ralentissant le processus de libéralisation économique et en fixant un cadre limité aux réformes institutionnelles.

\section{BIBLIOGRAPHIE}

Assemblée Nationale, Rapport d'information par la commission des affaires étrangères et la commission des finances en conclusion des travaux d'une mission d'information constituée le 3 février 2016 sur l'extraterritorialité de la législation américaine, 5 octobre 2016.

Cárdenas, Harold, La joven Cuba, https://jovencuba.com,10 janvier 2017.

Carranza, Julio, « La importancia del dato y la evidencia: algunas notas sobre el debate actual en Cuba », El estado como tal, https://elestadocomotal.com/, 8 juillet 2017.

Carranza, Julio, Luis Gutierrez y Pedro Monreal ,Cuba : la restructuracion de la economia, Talleres Gràficos, La Havane, 1996.

Castro , Fidel, Granma, http://www.granma.cu/, 28 mars 2016.

Castro Ruz, Raúl, Conclusions du 6e congrès du PCC, avril 2011.

Cuba Debate, « La economía cubana: actualizando el 2016 y una primera mirada al 2017 », juillet 2017.

Fernandez, Estrada y Julio Antonio, http://www.temas.cult.cu, page consultée le 18 septembre 2017, 
Howlett Martin, Patrick, « Dégel sous les tropiques », Le Monde diplomatique, Manière de voir, Octobre-novembre 2017.

Jolivet, Violaine, Miami la cubaine : géographie d'une ville carrefour entre les Amériques, Presses universitaires de Rennes, 2015.

Lequesne, Christian, Le Monde, 29/30 janvier 2017.

López Inguanzo, Joe Michel , La Joven Cuba, 17 novembre 2017.

Martinez Heredia, Francisco, www.cubadebate.cu, 28 août 2017.

Mendelson, Jean, « Cuba une place à part dans le monde depuis un demi-siècle », Questions Internationales, $\mathrm{n}^{\circ}$ 84, Mars/avril 2017.

Moreno Fraginals, Manuel, El Ingenio. Complejo económico-social cubano del azúcar, La Habana, Editorial de Ciencias Sociales, 1978.

Pañellas Álvarez, Daybel, "El otro polo de la desigualdad en Cuba: nuevos ricos, gerentes y famosos", TEMAS, n 84 ,Octobre/décembre 2015, http://www.temas.cult.cu, page consultée le 10 novembre 2017.

Perez, Humberto, "Principales debilidades internas actuales del modelo y perspectivas de nuestro socialismo", octobre 2016, https://elestadocomotal.com/, page consultée le 10 novembre 2017.

Rodriguez, José Luis, «La economia cubana , valoracion preliminar », Cuba Debate, août 2017, www.cubadebate.cu, page conseultée le 10 novembre 2017.

Valdès Paz, Juan , El Espacio y el limite, Ed. Ruth Casa Editorial, La Havane, 2009.

En savoir plus :

Juventud Rebelde : http://www.juventudrebelde.cu

14YMEDIO : www.14ymedio.com/

Cuba Posible : https://cubaposible.com/

Observatorio Critico : https://observatoriocriticocuba.org/

Havana Times : www.havanatimes.org/sp/

Noticias de Cuba : cubanet@cubanet.org

Diario de Cuba : www.diariodecuba.com/

Cubaencouentro: https://www.cubaencuentro.com/

\section{NOTES}

1. Cuban Liberty and Democratic Solidarity (Libertad) Act, 1996.

2. Commission for Assistance to a Free Cuba (CAFC). Elle a été créée par le Président George W. Bush le 10 Octobre 2003.

3. La loi est explicite : "The President is only permitted to "take steps to suspend the economic embargo" if a transitional government receives official recognition from Congress. Furthermore, a government in Cuba will not be considered "in transition to democracy" if Fidel or Raul Castro is in any way involved».

4. Deux caractérisations utilisées par les Cubains pour signifier la différence entre les deux politiques.

5. Concept développé par un universitaire américain, Joseph Nye.

6. L'embargo date de 1962.

7. D'où l'importance du marché noir et de l'économie informelle. 
8. «Conceptualización del modelo económico y social cubano de desarrollo socialista », 13 juillet 2017, www.cubadebate.cu.

9. Entretien oral avec l'auteur, mai 2016.

10. Il n'en est pas de même dans les médias et les journaux officiels.

11. Entretien oral avec l'auteur, mai 2016.

12. BioCubaFarma , 'Grupo de las Industrias Biotecnológica y Farmacéutica de Cuba' est une entreprise qui produit des médicaments, des équipements et des services de haute technologie. Avec plus de 21600 salariés, des centaines de spécialistes de haut niveau et 62 installations de production, c'est une industrie stratégique.

13. L'expression est de Violaine Jolivet.

14. Miami Herald, 17 juin 2014.

15. Témoignage de Leslie Thiercelin, septembre 2017, Paris, Mémoire de recherche sur la santé à Cuba.

16. «Los cambios institucionales que vendrán », 5 mai 2014.

17. La médiation de l'Eglise a également été utilisée par le gouvernement dans ses rapports avec la dissidence.

18. Il n'est pas possible dans le cadre de cet article d'analyser le poids très important des Forces Armées dans l'économie et le rôle d'une partie des militaires dans la gestion des entreprises.

19. Lors de la réunion El Último Jueves organisée à La Havane par la revue Temas, juin 2014.

\section{RÉSUMÉS}

L'amélioration des relations entre Cuba et les Etats-Unis est fondamentale pour des raisons géographiques, historiques et économiques. Les Etats-Unis sont le lieu de résidence de près de deux millions de Cubains émigrés, c'est le marché naturel qui convient à Cuba en raison de sa proximité, de son importance économique et commerciale. L'absence de relations normales avec les Etats-Unis, les sanctions économiques et commerciales et l'embargo imposé depuis plus d'un demi-siècle compromettent le développement économique du pays et sa stabilité sociale. Alors que le pays est engagé dans une transition difficile, le rétablissement des relations diplomatiques et la distension opérée sous la présidence Obama sont menacés par la présidence de Donald Trump.

The improvement of the bilateral relationship between the United States and Cuba is crucial for historical, geographical and economic reasons. The United States is now home to some two million people of Cuban origin. Due to its geographical proximity, its economic and commercial significance, it represents a perfect market for Cuba. The lack of normal relations with the United States, added to economic and commercial sanctions and a half-a-century old embargo, thwart the economic development of Cuba and its social stability. While the country is now involved in a difficult transition, the restoration of diplomatic relations and the thaw undertaken under the Obama Administration are now threatened by President Donald Trump.

La mejora de las relaciones entre Cuba y los Estados Unidos se debe fundamentalmente a razones geográficas, históricas y económicas. Los Estados Unidos es el lugar de residencia de dos millones de emigrantes cubanos y es un mercado natural conveniente para Cuba por su cercanía y su importancia económica y comercial. La ausencia de relaciones normales con los Estados Unidos, 
las sanciones económicas y comerciales así como el embargo impuesto desde hace más de medio siglo ponen en peligro el desarrollo económico del país y su estabilidad social. Mientras el país inicia una transición difícil, el restablecimiento de las relaciones diplomáticas y la distención operada durante la presidencia de Obama corren peligro bajo la presidencia de Donald Trump.

\section{INDEX}

Keywords : Normalization, embargo, reforms, transition, diaspora

Palabras claves : Normalización, embargo, reformas, transición, diáspora

Mots-clés : Normalisation, embargo, réformes, transition, diaspora

\section{AUTEUR}

\section{JANETTE HABEL}

Janette Habel est politiste, spécialiste de Cuba. Après avoir été maître de conférences à

l'Université Paris Est, elle est actuellement chercheur, animatrice du groupe de travail sur Cuba à l'Institut des Hautes Etudes de l'Amérique Latine (IHEAL) avec Stéphane Witkowski.janette.habel@wanadoo.fr 